This is the post-print version of the following article:

Ákos Kuki, Lajos Nagy, Miklós Zsuga and Sándor Kéki: Fast Identification of Phthalic Acid Esters in Poly(Vinyl Chloride) samples by Direct Analysis in Real Time (DART) Tandem Mass Spectrometry, International Journal of Mass Spectrometry, Volume 303, Issues 2-3, 1 June 2011, Pages 225-228

which has been published in final form at: http://dx.doi.org/10.1016/j.ijms.2011.02.011

\title{
Fast Identification of Phthalic Acid Esters in Poly(Vinyl Chloride) samples by Direct Analysis in Real Time (DART) Tandem Mass Spectrometry
}

\author{
Ákos Kuki, Lajos Nagy, Miklós Zsuga, Sándor Kéki* \\ Department of Applied Chemistry, University of Debrecen, H-4010 Debrecen, Hungary, \\ * Corresponding author: keki@tigris.unideb.hu, fax: +36 52 518662; H-4010 Debrecen, \\ HUNGARY
}

\begin{abstract}
It was found that the collision energy/voltage necessary to obtain $50 \%$ fragmentation $\left(\mathrm{CV}_{50}\right)$ was linearly dependent on the molecular weight of phthalic acid esters (PAEs). Based on this observation a fast screening technique for the detection of PAEs in Poly Vinyl Chloride (PVC) samples was developed using Direct Analysis in Real Time (DART) ionization tandem mass spectrometry. Based on this observation an automated data acquisition method, including mass-dependent tuning of the collision energy/voltage in DART-MS/MS, was developed thereby reducing the analysis time.
\end{abstract}

Keywords: phthalic acid ester, poly(vinyl chloride), Direct Analysis in Real Time ionization (DART), tandem mass spectrometry (MS/MS)

\section{Introduction}

Phthalic acid ester (PAE) plasticizers for poly(vinyl chloride) (PVC) are used to improve flexibility, workability and general handling properties. PAEs are utilized as plasticizers for the production of PVC goods including cords, films, toys, childcare articles and so forth. 
However, phthalate plasticizers are not bound covalently to the polymer matrix, therefore, they are able to migrate into the environment. Due to the potential risks of phthalates related to the health and the environment, regulation of PAE was issued by the European Parliament and the Council of the European Union [1] and by the US Congress [2]. With the introduction of a new ionization technique called Direct Analysis in Real Time (DART) it became possible to analyze ordinary objects in their native condition without time-consuming sample preparation steps [3, 4]. DART-MS has been successfully used to rapidly characterize surfaces of food contact substances and toys, their additives and contaminants, e.g. phthalates in PVC [5]. Moreover, in addition to DART-MS for the unambiguous structure identification of PAEs, DART combined with tandem mass spectrometry (DART-MS/MS) is highly desirable. To perform MS/MS the proper selection of collision energy is necessary for structure elucidation. Too low collision energy yields MS/MS spectra with very low intensity of the structurally important fragment ions, and at too high collision energy fragment ions resulting from high energy parallel/consecutive fragmentation steps may be present making spectral interpretation difficult. It is therefore essential for MS/MS measurements to set a proper collision energy/collision voltage to obtain reasonable fragmentation. In this work, we report a highly automated data acquisition method for DART-MS/MS, that is based on the mass-dependent setting of the collision energy/voltage for PAEs thereby reducing the analysis time to less than one minute per sample.

\section{Experimental}

\section{Chemicals}

HPLC grade methanol (Scharlau, Sentmenat, Spain) was used without further purification. Dibutyl phthalate (DBP), dihexyl phthalate (DHP), bis(2-ethylhexyl) phthalate (DEHP), di(nnonyl) phthalate (DNNP), diisononyl phthalate (DINP), octyl decyl phthalate (ODP), diisodecyl phthalate (DIDP), diundecyl phthalate (DUP), and ditridecyl phthalate (DTDP) were of technical grade and were provided by BorsodChem Ltd. (Kazincbarcika, Hungary). Six ordinary PVC objects were used for analysis: plug, watch band, certificate case, flooring, and a home-made PVC film were analyzed. The home-made PVC film was pressed from plasticized PVC granules provided by BorsodChem Ltd. (Kazincbarcika, Hungary) with warm press.

\section{Quadrupole Time-of-Flight Mass Spectrometry}


Measurements were performed with a MicroTOF-Q type Qq-TOF MS instrument from Bruker (Bruker Daltoniks, Bremen, Germany). For MS/MS experiments, nitrogen gas was used as the collision gas and the collision energies were varied in the range of 2-22 eV (in the laboratory frame). The pressure in the collision cell was determined to be $\sim 8 \times 10^{-3}$ mbar. The precursor ions for MS/MS were selected with an isolation width of 4 . All of the spectra were recorded by a digitizer at a sampling rate of $2 \mathrm{GHz}$. The accuracy of the $\mathrm{m} / \mathrm{z}$ determination was less than 0.01 in most cases. The mass spectra recorded were evaluated by the DataAnalysis 3.4 software from Bruker.

\section{Ion source for Direct Analysis in Real Time (DART)}

A DART SVP source was purchased from IonSense (IonSense, Inc., Saugus, MA, USA). The PVC samples were manually introduced into the DART gas stream. The gap between the ion source and the spectrometer inlet was $2.5 \mathrm{~cm}$. Samples were inserted into the middle of the gap. The DART system was operated in the positive mode at $250^{\circ} \mathrm{C}$ with helium 5.0 (purity $>$ $99.999 \%)$.

\section{Ion source for Atmospheric Pressure Chemical Ionization (APCI)}

Atmospheric Pressure Chemical Ionization (APCI) source was from Bruker (Bruker Daltoniks, Bremen, Germany). The PAE standard samples were dissolved in methanol at a concentration of $1 \mathrm{mg} / \mathrm{mL}$. The PAE solutions were delivered directly into the APCI source with a syringe pump (Cole-Parmer Ins. Co., Vernon Hills, IL, USA) at a flow rate of 15 $\mu \mathrm{L} / \mathrm{min}$ together with a carrier flow of methanol at a flow rate of $0.2 \mathrm{~mL} / \mathrm{min}$ by means of a T-piece.

\section{Evaluation of the survival yield curves}

The efficiency of the fragmentation can be described quantitatively by the survival yield (SY) [6-8]. The SY is defined according to eq 1:

$$
S Y=\frac{I_{p}}{I_{p}+\sum I_{f}}
$$

where $\mathrm{I}_{\mathrm{p}}$ is the intensity of the precursor ion, and $\Sigma \mathrm{I}_{\mathrm{f}}$ is the sum of all fragment ion intensities. 
The shape of the SY curve is a sigmoid type and can be described by a two-parameter sigmoid function according to eq 2 :

$$
\mathrm{SY}=\frac{1}{1+\mathrm{a} e^{\mathrm{bCV}}}
$$

where $\mathrm{a}$ and $\mathrm{b}$ are constants for each compound and were determined by fitting to the experimental data, and $\mathrm{CV}$ is the collision voltage.

For fitting the parameters of the two-parameter sigmoid function (eq 2) to the experimental data, a home-made software utilizing the Gauss-Newton-Marquardt procedure was applied [9]. The collision voltage $\left(\mathrm{CV}_{50}\right)$ at which the intensity of the precursor ion is equal to that of all fragment ions, i.e., at $50 \%$ fragmentation $(\mathrm{SY}=0.5)$ was determined by eq 3 .

$$
\mathrm{CV}_{50}=\frac{-\ln (\mathrm{a})}{\mathrm{b}}
$$

\section{Results and Discussion}

Usually some very intense peaks appeared after positioning a PVC sample in the DART source. These peaks can be assigned to the phthalic acid ester plasticizers. As a representative example, Fig. 1 shows the DART MS spectrum of a certificate case. In the low $\mathrm{m} / \mathrm{z}$ region the most intense peaks belong to the protonated dioctyl phthalate (DOP), m/z 391 and didecyl phthalate (DDP), $\mathrm{m} / \mathrm{z} 447$. For both PAEs the ammonium adducts can also be detected $(\mathrm{m} / \mathrm{z}$ 408 and 464 for DOP and DDP, respectively) with different intensities of about $30-40 \%$. The three intense peaks in the high $\mathrm{m} / \mathrm{z}$ region of the MS spectrum were assigned to the ammonium ion adducts of the DOP and DDP dimers at $\mathrm{m} / \mathrm{z} 798$ and 910, respectively and the mixed DOP-DDP dimer at $\mathrm{m} / \mathrm{z} 854$.

Fig. 1.

However, based on only the MS spectra PAE isomers cannot be distinguished, MS/MS experiments are needed for the unambiguous PEA identification. For example, dinonyl phthalate (DNP) has the same elemental composition as that of octyl decyl phthalate (ODP) $\left(\mathrm{C}_{26} \mathrm{H}_{42} \mathrm{O}_{4}\right)$, hence the peak at $\mathrm{m} / \mathrm{z} 419$ can be due to either the protonated DNP or ODP. Therefore, these PAE isomers should be differentiated by MS/MS examination of their characteristic fragment ions. 
Therefore, to perform a fast qualitative analysis of PVC samples the acquisition and procession of the MS and MS/MS spectra must be automated. For this reason we developed a short software module which is based on two key issues: the selection of the proper collision voltage and identification of PAEs based on the characteristic fragments formed from protonated precursors.

\section{Selection of the proper collision energy/voltage for MS/MS}

For obtaining useful MS/MS spectra the selection of the proper collision energy/voltage is also critical since the collision voltage should be varied as the mass of the PAEs changes. Therefore, we examined the relationship between the collision voltage necessary to achieve $50 \%$ fragmentation $\left(\mathrm{CV}_{50}\right)$ by means of the survival yield curves (SY) and the mass of the PAEs. Some representative examples of SY curves are shown in Fig. 2.

Fig. 2.

The SY curves were determined from the APCI-MS/MS spectra of the PAEs. The source conditions of APCI were similar to those of DART, therefore the results obtained from APCIMS/MS can be applied to the SY of DART-MS/MS. This was confirmed by comparing some SY values obtained from APCI-MS/MS to those by DART-MS/MS. For example, the SY of DEHP at collision voltage $8 \mathrm{~V}$ obtained from APCI and DART is 0.3 and 0.25 , respectively. Fig. 3. shows the variation of $\mathrm{CV}_{50}$ with the mass of PAEs.

Fig. 3.

As seen in Fig. 3. a linear relationship was found between $\mathrm{CV}_{50}$ and the mass of PAEs allowing an easy setting of the collision voltage for the PAEs.

\section{Characteristic peaks of PAEs for automated recognition}

For the automated recognition of the different PAEs usually three peaks were selected in the case of each PAEs. The first characteristic peak is the protonated PAE molecule itself which is the precursor ion of the MS/MS analysis. The second is the typical protonated fragment ion of PAEs at $\mathrm{m} / \mathrm{z} 149$, and the third is the fragment ion formed by the loss of an alkene or a fatty alcohol group, e.g. octene or octanol for DOP. Furthermore, it is also possible to differentiate di(n-octyl) phthalate (DNOP) and DEHP isomers based on the intensity ratio of the fragment 
ions formed by the loss of alkene and fatty alcohol units [5]. Table 1 summarizes the characteristic fragment ions used for the identification of PAEs.

Table 1.

\section{Developing an automated method for PAEs identification}

On these basis we developed a control and evaluation method using the Auto MSMS feature of the Bruker acquisition software for the automatic recognition, isolation and fragmentation. The evaluation of the MS/MS data requires an automated post-run data processing too, so the control method was extended with a data analysis part (a home made software module developed in Visual Basic Script language which is appended as supplementary data). For example, the analysis of the watch band (one of our test samples) was performed as follows: the acquisition software controlled by our method works in the AutoMSMS mode. The spectrometer first scans in MS mode and finds two precursor ions which are included in the list of the possible protonated PAE compounds at $\mathrm{m} / \mathrm{z} 391$ as potential DOP and $\mathrm{m} / \mathrm{z} 419$ as potential DNP or ODP. After the short MS segment the spectrometer automatically switches to MS/MS mode and subsequently set the isolation mass to $m / z 391$ and 419. The collision voltage for each precursor ion is stored in the method file which has been obtained previously from the $\mathrm{CV}_{50}-\mathrm{m} / z$ curves. For the post-run processing the raw data files are opened with the data analysis software and evaluated by the processing software module. The software creates two MS/MS spectra with the precursor ions $\mathrm{m} / z 391$ and 419 and identifies DEHP based on the characteristic fragment peaks of the first MS/MS spectrum, and DINP based on the characteristic fragment peaks of the second MS/MS spectrum. The PAEs identified in the six PVC objects are compiled in Table 2.

Table 2.

The average measurement time of a sample including data acquisition and post processing was less than one minute.

\section{Conclusions}

Utilizing tandem mass spectrometry with an ambient desorption ionization technique and applying automated data acquisition and processing methods a very short analysis time (less than one minute) was achieved for the qualitative determination of the PAE plasticizer- 
content in PVC objects. A linear relationship was found between the collision energy necessary to obtain 50\% fragmentation and the mass of PAEs allowing an easy tuning of the instrument voltage to achieve appropriate fragmentation extent for PAE-identification. In addition, due to the linearity of the $\mathrm{CV}_{50}-\mathrm{m} / \mathrm{z}$ curve, a quick, two-point calibration can be applied to set the appropriate collision voltages for PAEs with different masses. Furthermore, our plasticizer scanning technique presented in this article can also be extended to other PAEs.

\section{Acknowledgment}

This work was financially supported by the grant K-72524 given by OTKA (National Found for Scientific Research Development, Hungary), and the grants GVOP-3.2.1.-2004-040152/3.0. and TAMOP 4.2.1./B-09/1/KONV-2010-0007. 


\section{References}

[1] European Communities. Directive 2005/84/EC of the European Parliament and of the Council. Off. J. Eur. Union (2005) L 344:40.

[2] 110th United States Congress. Consumer Product Safety Improvement Act (CPSIA), (2008), Available: http://www.cpsc.gov/cpsia.pdf

[3] R.B. Cody, J.A. Laramee, H.D. Durst, Versatile new ion source for the analysis of materials in open air under ambient conditions, Anal. Chem. 77 (2005) 2297-2302.

[4] R.B. Cody, J.A. Laramee, J.M. Nilles, H.D. Durst, Direct Analysis in Real Time $\left(\right.$ DART $\left.^{\mathrm{tm}}\right)$ Mass Spectrometry, JEOL News 40 (2005) 8-12.

[5] T. Rothenbacher, W. Schwack, Rapid and nondestructive analysis of phthalic acid esters in toys made of poly(vinyl chloride) by direct analysis in real time single-quadrupole mass spectrometry, Rapid Commun. Mass Spectrom. 23 (2009) 2829-2835.

[6] F. Derwa, E.D. Pauw, P. Natalis, New basis for a method for the estimation of secondary ion internal energy-distribution in soft ionization techniques, Org. Mass Spectrom. 26 (1991) 117-118.

[7] X.H. Guo, M.C. Duursma, P.G. Kistemaker, N.M.M. Nibbering, K. Vekey, L. Drahos, R.M. Heeren, Manipulating internal energy of protonated biomolecules in electrospray ionization Fourier transform ion cyclotron resonance mass spectrometry, J. Mass Spectrom. 38 (2003) 597-606.

[8] A. Memboeuf, A. Nasioudis, S. Indelicato, F. Pollreisz, Á. Kuki, S. Kéki, O.F. van den Brink, K. Vékey, L. Drahos, Size effect on fragmentation in tandem mass spectrometry, Anal. Chem. 82 (2010) 2294-2302.

[9] D. Marquardt, An Algorithm for Least-Squares Estimation of Nonlinear Parameters, SIAM J. Appl. Math. 11 (1963) 431-441. 


\section{Legends for the Figures}

Fig. 1.

DART MS spectrum of an old PVC certificate case.

Fig. 2.

Survival yield (SY) versus collision voltage (CV) curves for DBP, DNP and DUP. The solid lines represent the fitted curves by eq 2 . The fitted parameters are: $a=0.040$ and $b=0.651$ for DBP, $a=9.34 \times 10^{-3}$ and $b=0.603$ for DNP and $a=3.17 \times 10^{-3}$ and $b=0.619$ for DUP.

\section{Fig. 3.}

Variation of the $\mathrm{CV}_{50}$ value with $\mathrm{m} / \mathrm{z}$ for the PAEs (filled circles). The linear correlation between the $\mathrm{CV}_{50}$ and $\mathrm{m} / \mathrm{z}$ is presented as a solid line. 
Table 1. The $m / z$ values of the characteristic fragment peaks used for PAE-identification. The column on the right gives criteria for distinguishing between the isomers.

\begin{tabular}{|c|c|c|c|c|c|}
\hline & \multicolumn{4}{|c|}{ Characteristic fragment ions } & \multirow{2}{*}{$\begin{array}{c}\text { Differentiation between } \\
\text { isomers }\end{array}$} \\
\hline & $\underline{1}$ & $\underline{2}$ & $\underline{\mathbf{3}}$ & $\underline{4}$ & \\
\hline DHP & 335 & 149 & 233 & 251 & \\
\hline $\begin{array}{l}\text { DNOP } \\
\text { DEHP }\end{array}$ & 391 & 149 & 261 & 279 & $\begin{array}{l}\text { intensity }_{261}>\text { intesity }_{279} \\
\text { intensity }{ }_{261}<\text { intesity }_{279}\end{array}$ \\
\hline $\begin{array}{l}\text { DNNP } \\
\text { DINP }\end{array}$ & 419 & 149 & 275 & 293 & $\begin{array}{l}\text { intensity }_{275}>10^{*} \text { intesity }_{293} \\
\text { intensity }_{275}<10 * \text { intesity }_{293}\end{array}$ \\
\hline ODP* & 419 & 149 & 261 & 279 & \\
\hline DIDP & 447 & 149 & 289 & 307 & \\
\hline DUP & 475 & 149 & 303 & 321 & \\
\hline DTDP & 531 & 149 & 331 & 349 & \\
\hline
\end{tabular}

$\underline{1}$ Precursor ion $\underline{\mathbf{2}}$ fragment ion formed by the losses of alkene $\left(\mathrm{C}_{n} \mathrm{H}_{2 n}\right)$ and fatty alcohol $\left(\mathrm{C}_{n} \mathrm{H}_{2 n+1} \mathrm{OH}\right)$ units $\underline{\mathbf{3}}$ fragment ion formed by the loss of fatty alcohol $\underline{\mathbf{4}}$ fragment ion formed by the loss of alkene unit.

*in the case of ODP additional fragment ions at m/z 307 and 289 formed by the loss of $\mathrm{C}_{8} \mathrm{H}_{16}$ and $\mathrm{C}_{8} \mathrm{H}_{17} \mathrm{OH}$ units, respectively were taken into account.

Table 2. The identified PAE compounds in the PVC objects.

\begin{tabular}{ccc}
\hline & PAE 1 & PAE 2 \\
\hline PVC film & DEHP & - \\
plug & DINP & - \\
watch band & DEHP & DINP \\
certificate case & DEHP & DIDP \\
PVC flooring & DEHP & DINP \\
PVC glasses & DEHP & - \\
\hline
\end{tabular}


Fig. 1.

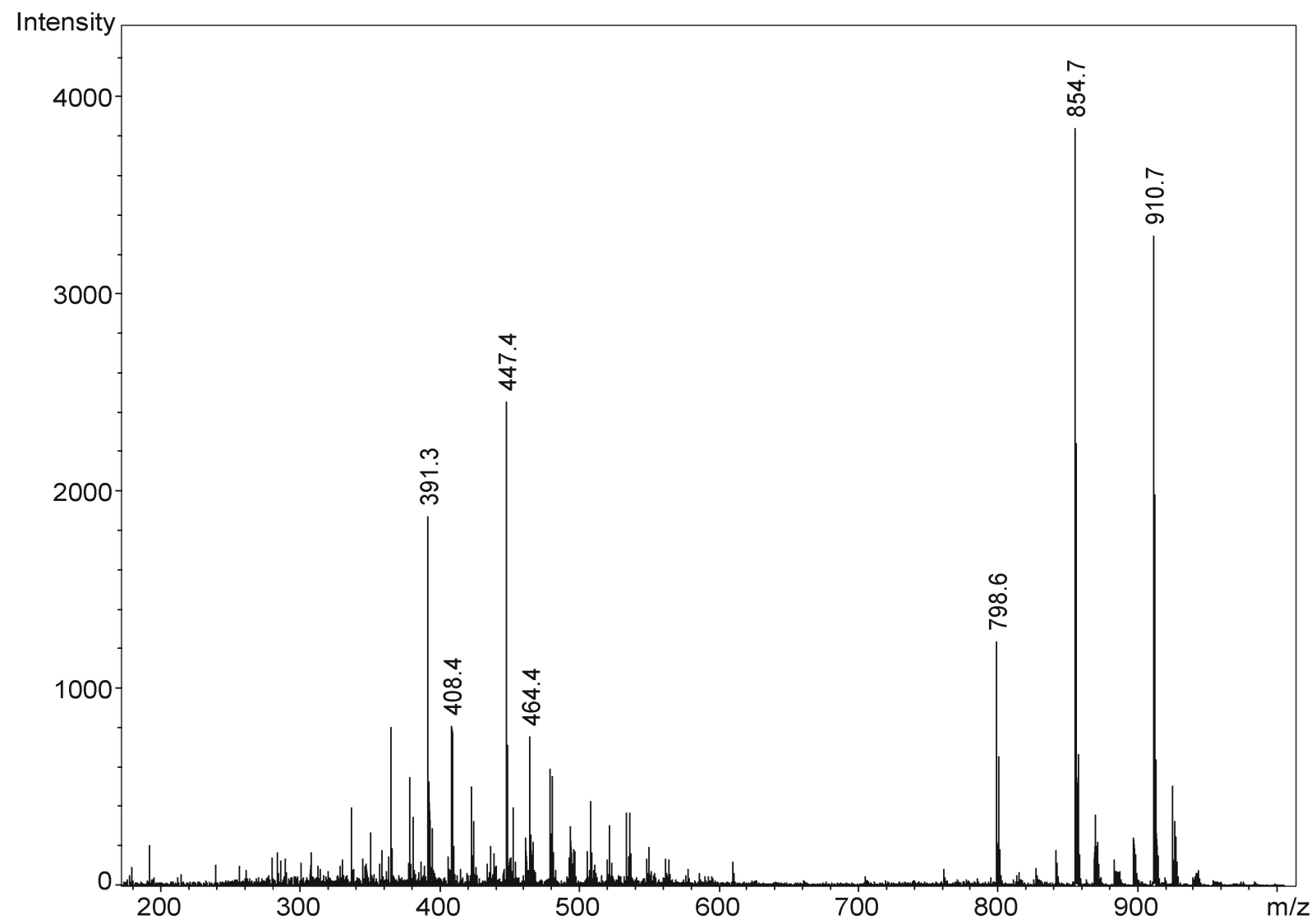


Fig. 2.

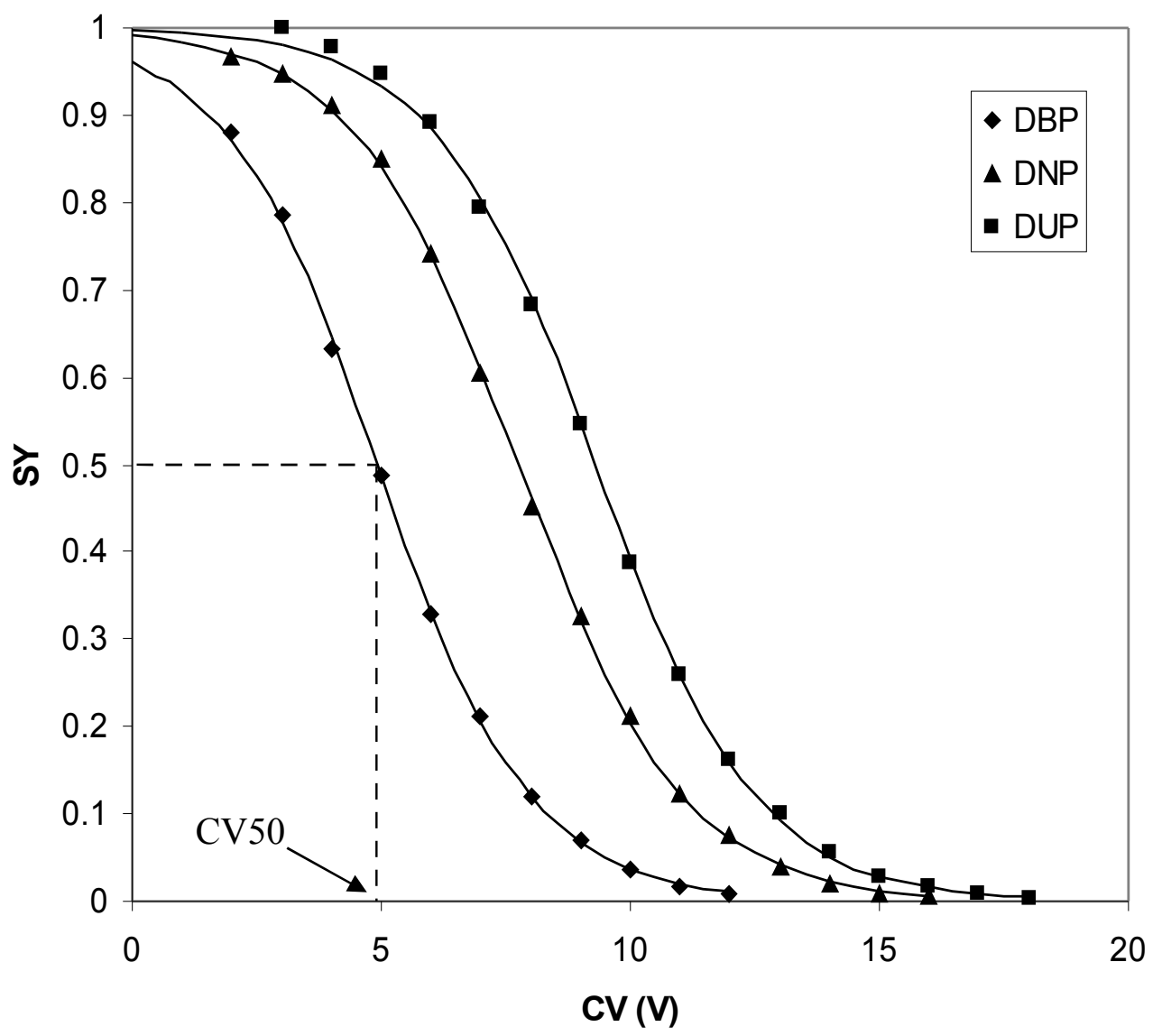


Fig. 3.

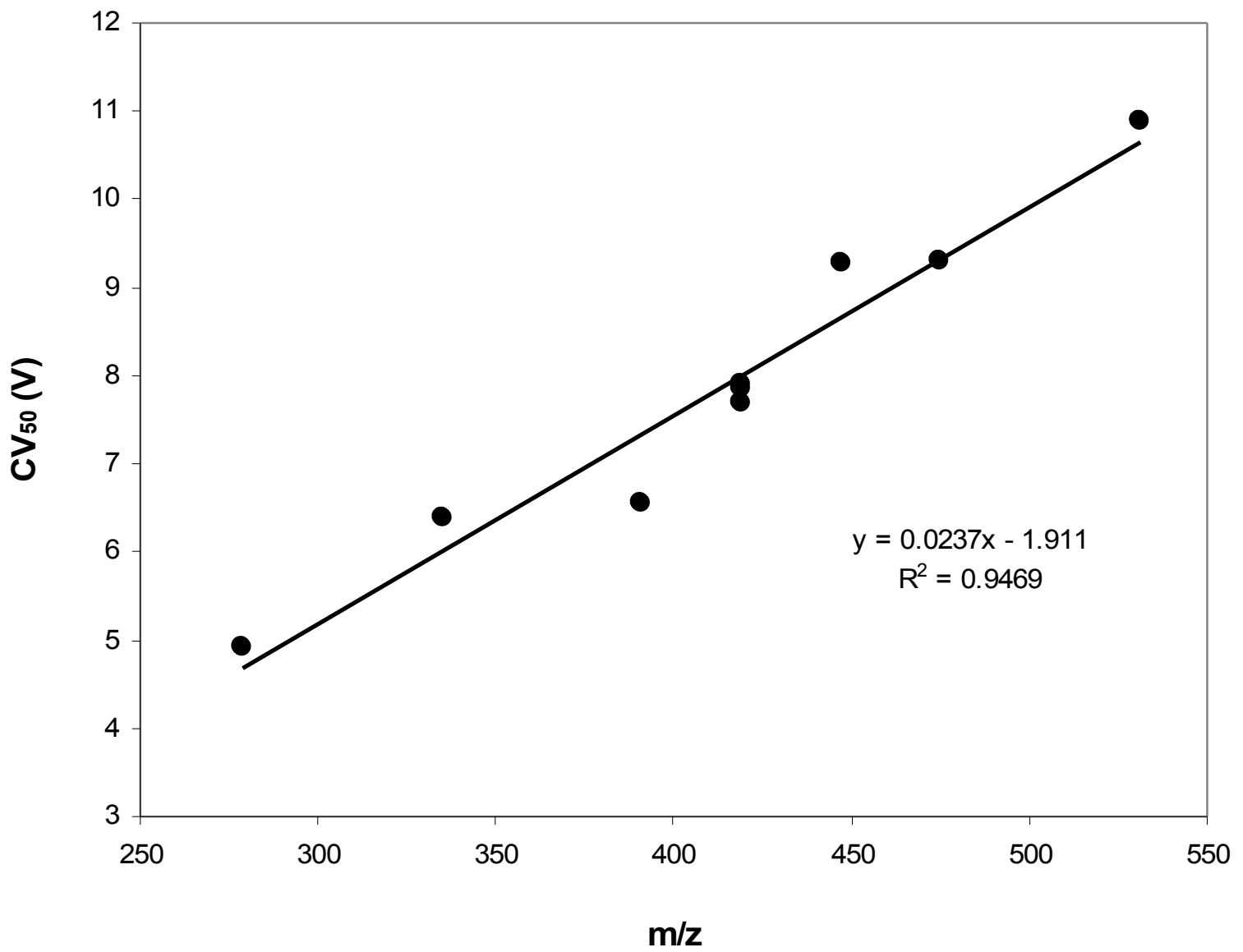

